Acta Crystallographica Section E

Structure Reports

Online

ISSN 1600-5368

\section{4-Ethoxy- $N^{\prime}$-propanoylpyridine-2-carbo- hydrazide}

\section{Sheng-Jiao Tang, Hai-Ping Li, Xin-Yong Lin and Wen-Shi Wu*}

College of Materials Science and Engineering, Huaqiao University, Xiamen, Fujian 361021, People's Republic of China

Correspondence e-mail: wws@hqu.edu.cn

Received 6 March 2010; accepted 18 March 2010

Key indicators: single-crystal X-ray study; $T=293 \mathrm{~K}$; mean $\sigma(\mathrm{C}-\mathrm{C})=0.003 \AA$; $R$ factor $=0.053 ; w R$ factor $=0.150 ;$ data-to-parameter ratio $=18.2$.

In the crystal structure of the title compound, $\mathrm{C}_{11} \mathrm{H}_{15} \mathrm{~N}_{3} \mathrm{O}_{3}$, molecules are linked into a chain by intermolecular $\mathrm{N}-\mathrm{H} \cdots \mathrm{O}$ hydrogen bonds.

\section{Related literature}

For the structure of $N$-propionylpicoloylhydrazide, see: Wu \& Liu (2001).<smiles>CCOc1ccnc(C(=O)NNC(=O)CC)c1</smiles>

\section{Experimental}

Crystal data

$$
\begin{aligned}
& \mathrm{C}_{11} \mathrm{H}_{15} \mathrm{~N}_{3} \mathrm{O}_{3} \\
& M_{r}=237.26 \\
& \text { Monoclinic, } P 2_{1} / c
\end{aligned}
$$

$\beta=99.534(5)^{\circ}$

$V=1237.3(9) \AA^{3}$

$Z=4$

Mo $K \alpha$ radiation

$\mu=0.09 \mathrm{~mm}^{-1}$

$T=293 \mathrm{~K}$

$1.00 \times 0.45 \times 0.10 \mathrm{~mm}$

Data collection

Bruker P4 diffractometer

9032 measured reflections

2803 independent reflections

2297 reflections with $I>2 \sigma(I)$

$R_{\text {int }}=0.022$

Refinement

$R\left[F^{2}>2 \sigma\left(F^{2}\right)\right]=0.053$

$w R\left(F^{2}\right)=0.150$

$S=0.96$

2803 reflections

154 parameters

$\mathrm{H}$-atom parameters constrained

$\Delta \rho_{\max }=0.27{\mathrm{e} \AA^{-3}}^{\circ}$

$\Delta \rho_{\min }=-0.28$ e $\AA^{-3}$

Table 1

Hydrogen-bond geometry $\left(\AA,^{\circ}\right)$.

\begin{tabular}{lllll}
\hline$D-\mathrm{H} \cdots A$ & $D-\mathrm{H}$ & $\mathrm{H} \cdots A$ & $D \cdots A$ & $D-\mathrm{H} \cdots A$ \\
\hline $\mathrm{N} 2-\mathrm{H} 2 A \cdots \mathrm{O} 2^{\mathrm{i}}$ & 0.86 & 2.43 & $3.067(2)$ & 132 \\
$\mathrm{~N} 3-\mathrm{H} 3 A \cdots \mathrm{O} 2^{\mathrm{ii}}$ & 0.86 & 2.06 & $2.831(2)$ & 150 \\
\hline
\end{tabular}

Symmetry codes: (i) $-x, y+\frac{1}{2},-z+\frac{1}{2}$; (ii) $x, y+1, z$.

Data collection: XSCANS (Bruker, 1999); cell refinement: XSCANS; data reduction: SHELXTL (Sheldrick, 2008); program(s) used to solve structure: SHELXS97 (Sheldrick, 2008); program(s) used to refine structure: SHELXL97 (Sheldrick, 2008); molecular graphics: SHELXTL; software used to prepare material for publication: SHELXTL.

We are grateful for financial support from the National Science Foundation of Fujian Province of China (No. E0610017, 2003 F006).

Supplementary data and figures for this paper are available from the IUCr electronic archives (Reference: JH2133).

\title{
References
}

Bruker (1999). XSCANS. Bruker AXS Inc., Madison, Wisconsin, USA. Sheldrick, G. M. (2008). Acta Cryst. A64, 112-122.

Wu, W. S. \& Liu, S. X. (2001). Chin. J. Struct. Chem. 20, 226-228. 


\section{supporting information}

Acta Cryst. (2010). E66, o920］[doi:10.1107/S1600536810010135]

\section{4-Ethoxy-N'-propanoylpyridine-2-carbohydrazide}

\section{Sheng-Jiao Tang, Hai-Ping Li, Xin-Yong Lin and Wen-Shi Wu}

\section{S1. Comment}

It has been reported that the structure of N-propionylpicoloylhydrazide (II, Wu et al., 2001), based on which we reported the structure of 4-ethoxy- $N$-propionyl-2-pyridine formylhydrazine (I), $\mathrm{C}_{11} \mathrm{H}_{15} \mathrm{~N}_{3} \mathrm{O}_{3}$. The structure of the title compound shown in Fig. 1 exhibits a stable one-dimension chain structure which is stabilized by inter-molecular hydrogen bonds of $\mathrm{N} 2-\mathrm{H} 2 \mathrm{~A} \cdots \mathrm{O} 2^{\mathrm{i}}, \mathrm{N} 3-\mathrm{H} 3 \mathrm{~A} \cdots \mathrm{O} 2^{\mathrm{ii}}$. All these bonds are detailed in Fig. 2 and Table 1.

In this title compound, the torsion angle of $\mathrm{C} 6-\mathrm{N} 2-\mathrm{N} 3-\mathrm{C} 7$ is -77.1 (2) ${ }^{\circ}$ which is slightly smaller than the torsion angle of the structure (II) $\left(-73.5(2)^{\circ}\right.$ for $\left.\mathrm{C} 6-\mathrm{N} 2-\mathrm{N} 3-\mathrm{C} 7\right)$. The distances of $\mathrm{C} 6-\mathrm{N} 2, \mathrm{~N} 2-\mathrm{N} 3$ and $\mathrm{C} 7-\mathrm{N} 3$ are 1.345 (2) $\AA, 1.335$ (2) and 1.380 (2) $\AA$ respectively. They are similar to homologous bonds of structure (II) with distances 1.334 (2) $\AA$ for $\mathrm{C} 6-\mathrm{N} 2,1.383$ (2) $\AA$ for N2-N3 and 1.337 (2) $\AA$ for C7-N3. And in structure (I), it's almost coplanar for $\mathrm{C} 1, \mathrm{C} 2, \mathrm{C} 3, \mathrm{C} 4, \mathrm{C} 5, \mathrm{~N} 1, \mathrm{C} 6, \mathrm{O} 1, \mathrm{~N} 2, \mathrm{~N} 3, \mathrm{O} 3, \mathrm{C} 10$ and C11, and the maximum atomic deviation being $0.0920 \AA$. The dihedral angle between the mean planes of the $\mathrm{C} 1, \mathrm{C} 2, \mathrm{C} 3, \mathrm{C} 4, \mathrm{C} 5, \mathrm{~N} 1, \mathrm{C} 6, \mathrm{O} 1, \mathrm{~N} 2, \mathrm{~N} 3, \mathrm{O} 3, \mathrm{C} 10$ and $\mathrm{C} 11$ and the mean planes of the N3, C7 and $\mathrm{O} 2$ is $72.44(8)^{\circ}$.

\section{S2. Experimental}

4-ethoxyl-2-pyridine formylhydrazine $(3.42 \mathrm{~g}, 14.41 \mathrm{mmol})$ was dissolved in a mixed solution of $\mathrm{CHCl}_{3}(30 \mathrm{ml})$ and EtOH $(20 \mathrm{ml})$, then filtered. Propionic anhydride $(3.64 \mathrm{ml})$ was added and refluxed $2 \mathrm{hrs}$ with whisked. Colorless needle crystals of the title compound were obtained by slow evaporation of solvent at room temperature. Melting point: 407 $407.5 \mathrm{~K}$.

\section{S3. Refinement}

The positions of the N2-, N3-bound $\mathrm{H}$ atoms were placed at fixed positions and refined accord to the riding model. The $\mathrm{C}$-bound $\mathrm{H}$ atoms were included in the riding model approximation with $\mathrm{C}-\mathrm{H}=0.93 \AA$ and $\mathrm{U}_{\text {iso }}$ of each $\mathrm{H}$ atom $=$ $1.2 \mathrm{U}_{\mathrm{eq}}(\mathrm{C})$. 


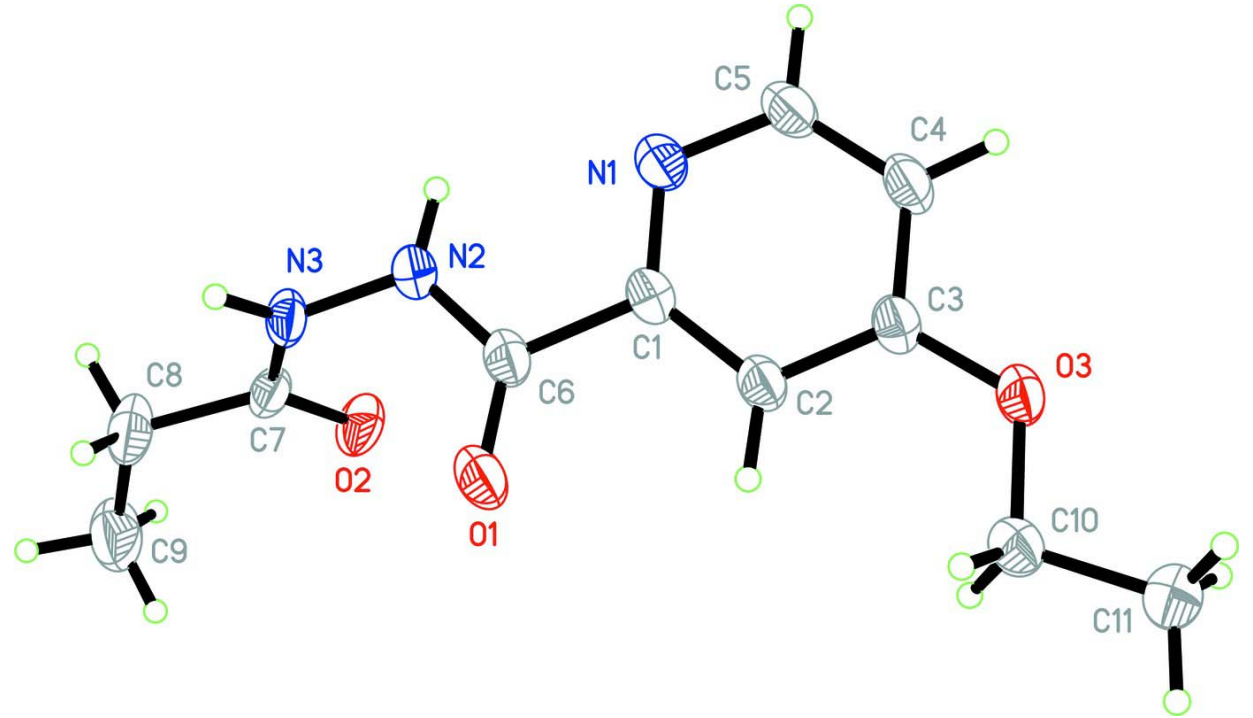

\section{Figure 1}

View of the title compound showing the atom-labelling scheme. Displacement ellipsoids are drawn at the $30 \%$ probability level. $\mathrm{H}$ atoms are represented by circles of arbitrary radius. 


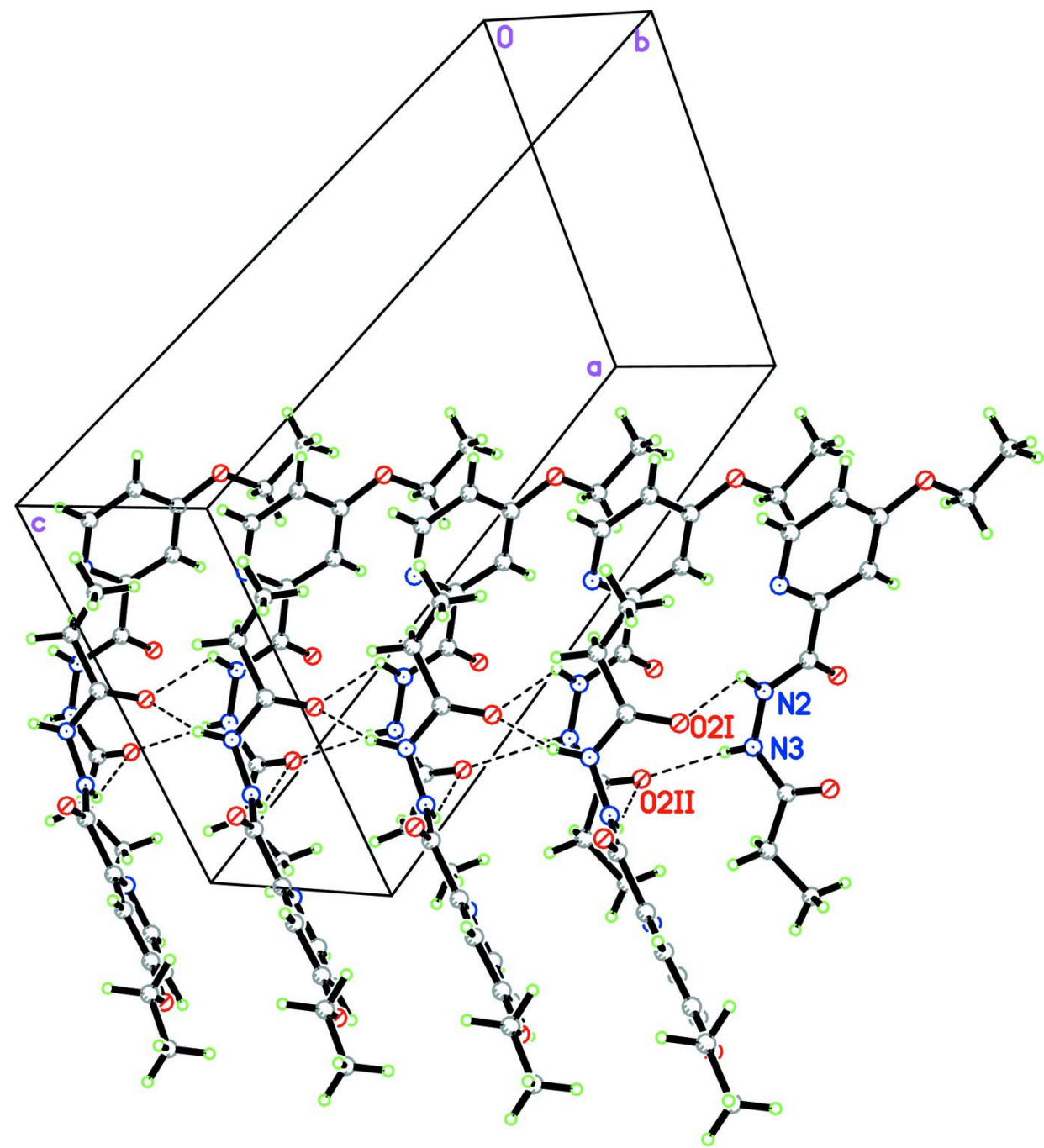

Figure 2

Hydrogen bonds diagram of the title compound, showing the H-bonded interactions (dashed lines). O2I, O2II represent $\mathrm{O} 2^{\mathrm{i}}, \mathrm{O} 2^{\mathrm{ii}}$, respectively.

\section{4-Ethoxy- $N^{\prime}$-propanoylpyridine-2-carbohydrazide}

Crystal data

$\mathrm{C}_{11} \mathrm{H}_{15} \mathrm{~N}_{3} \mathrm{O}_{3}$

$M_{r}=237.26$

Monoclinic, $P 2_{1} / c$

Hall symbol: -P 2ybc

$a=11.377(5) \AA$

$b=4.745(2) \AA$

$c=23.244(10) \AA$

$\beta=99.534(5)^{\circ}$

$V=1237.3(9) \AA^{3}$

$Z=4$
$F(000)=504$

$D_{\mathrm{x}}=1.274 \mathrm{Mg} \mathrm{m}^{-3}$

Melting point $=407-407.5 \mathrm{~K}$

Mo $K \alpha$ radiation, $\lambda=0.71073 \AA$

Cell parameters from 2827 reflections

$\theta=2.7-27.5^{\circ}$

$\mu=0.09 \mathrm{~mm}^{-1}$

$T=293 \mathrm{~K}$

Prism, colourless

$1.00 \times 0.45 \times 0.10 \mathrm{~mm}$ 


\section{Data collection}

Bruker P4

diffractometer

Radiation source: fine-focus sealed tube

Graphite monochromator

Detector resolution: 0 pixels $\mathrm{mm}^{-1}$

$\omega$ scans

9032 measured reflections

\section{Refinement}

Refinement on $F^{2}$

Least-squares matrix: full

$R\left[F^{2}>2 \sigma\left(F^{2}\right)\right]=0.053$

$w R\left(F^{2}\right)=0.150$

$S=0.96$

2803 reflections

154 parameters

0 restraints

Primary atom site location: structure-invariant direct methods
2803 independent reflections

2297 reflections with $I>2 \sigma(I)$

$R_{\text {int }}=0.022$

$\theta_{\max }=27.5^{\circ}, \theta_{\min }=2.7^{\circ}$

$h=-14 \rightarrow 11$

$k=-6 \rightarrow 6$

$l=-24 \rightarrow 30$

Secondary atom site location: difference Fourier map

Hydrogen site location: inferred from neighbouring sites

$\mathrm{H}$-atom parameters constrained

$w=1 /\left[\sigma^{2}\left(F_{\mathrm{o}}{ }^{2}\right)+(0.080 P)^{2}+0.3962 P\right]$

where $P=\left(F_{\mathrm{o}}{ }^{2}+2 F_{\mathrm{c}}{ }^{2}\right) / 3$

$(\Delta / \sigma)_{\max }<0.001$

$\Delta \rho_{\max }=0.27 \mathrm{e} \AA^{-3}$

$\Delta \rho_{\min }=-0.28$ e $\AA^{-3}$

Special details

Geometry. All esds (except the esd in the dihedral angle between two 1.s. planes) are estimated using the full covariance matrix. The cell esds are taken into account individually in the estimation of esds in distances, angles and torsion angles; correlations between esds in cell parameters are only used when they are defined by crystal symmetry. An approximate (isotropic) treatment of cell esds is used for estimating esds involving l.s. planes.

Refinement. Refinement of $F^{2}$ against ALL reflections. The weighted $R$-factor wR and goodness of fit $S$ are based on $F^{2}$, conventional $R$-factors $R$ are based on $F$, with $F$ set to zero for negative $F^{2}$. The threshold expression of $F^{2}>\sigma\left(F^{2}\right)$ is used only for calculating $R$-factors (gt) etc. and is not relevant to the choice of reflections for refinement. $R$-factors based on $F^{2}$ are statistically about twice as large as those based on $F$, and $R$ - factors based on ALL data will be even larger.

Fractional atomic coordinates and isotropic or equivalent isotropic displacement parameters $\left(\AA^{2}\right)$

\begin{tabular}{lllll}
\hline & $x$ & $y$ & $z$ & $U_{\text {iso }} * / U_{\text {eq }}$ \\
\hline C1 & $0.16780(13)$ & $0.4979(3)$ & $0.41169(7)$ & $0.0451(4)$ \\
C2 & $0.18448(13)$ & $0.3174(3)$ & $0.45907(7)$ & $0.0476(4)$ \\
H2 & 0.1211 & 0.2618 & 0.4771 & $0.057^{*}$ \\
C3 & $0.29993(13)$ & $0.2221(4)$ & $0.47874(6)$ & $0.0467(4)$ \\
C4 & $0.39038(13)$ & $0.3146(4)$ & $0.45039(7)$ & $0.0552(4)$ \\
H4 & 0.4682 & 0.2531 & 0.4622 & $0.066^{*}$ \\
C5 & $0.36375(14)$ & $0.4981(4)$ & $0.40462(8)$ & $0.0608(5)$ \\
H5 & 0.4260 & 0.5619 & 0.3867 & $0.073^{*}$ \\
C6 & $0.04284(13)$ & $0.5972(4)$ & $0.38881(7)$ & $0.0493(4)$ \\
C7 & $-0.15075(13)$ & $0.6370(3)$ & $0.27838(7)$ & $0.0446(4)$ \\
C8 & $-0.26718(18)$ & $0.7610(4)$ & $0.24930(10)$ & $0.0732(6)$ \\
H8A & -0.2938 & 0.8948 & 0.2760 & $0.088^{*}$ \\
H8B & -0.2530 & 0.8654 & 0.2152 & $0.088^{*}$ \\
C9 & $-0.36264(19)$ & $0.5625(6)$ & $0.23120(15)$ & $0.1004(9)$ \\
H9D & -0.4327 & 0.6624 & 0.2134 & $0.151^{*}$ \\
H9A & -0.3795 & 0.4607 & 0.2646 & $0.151^{*}$ \\
H9B & -0.3391 & 0.4325 & 0.2036 & $0.151^{*}$
\end{tabular}




$\begin{array}{lllll}\text { C10 } & 0.24191(15) & -0.0530(4) & 0.55627(8) & 0.0557(4) \\ \text { H10A } & 0.1822 & -0.1624 & 0.5311 & 0.067^{*} \\ \text { H10B } & 0.2029 & 0.1047 & 0.5718 & 0.067^{*} \\ \text { C11 } & 0.30482(18) & -0.2325(5) & 0.60472(9) & 0.0708(6) \\ \text { H11A } & 0.2481 & -0.3041 & 0.6274 & 0.106^{*} \\ \text { H11B } & 0.3636 & -0.1216 & 0.6292 & 0.106^{*} \\ \text { H11C } & 0.3431 & -0.3871 & 0.5886 & 0.106^{*} \\ \text { N1 } & 0.25346(11) & 0.5917(3) & 0.38402(6) & 0.0540(4) \\ \text { N2 } & 0.03317(11) & 0.7407(3) & 0.33833(6) & 0.0506(3) \\ \text { H2A } & 0.0960 & 0.7822 & 0.3240 & 0.061^{*} \\ \text { N3 } & -0.07761(11) & 0.8213(3) & 0.30948(6) & 0.0490(3) \\ \text { H3A } & -0.0999 & 0.9937 & 0.3116 & 0.059^{*} \\ \text { O1 } & -0.04003(11) & 0.5488(4) & 0.41406(6) & 0.0787(5) \\ \text { O2 } & -0.12542(11) & 0.3873(2) & 0.27472(6) & 0.0604(4) \\ \text { O3 } & 0.33191(10) & 0.0459(3) & 0.52425(5) & 0.0598(4)\end{array}$

Atomic displacement parameters $\left(\AA^{2}\right)$

\begin{tabular}{lllllll}
\hline & $U^{11}$ & $U^{22}$ & $U^{33}$ & $U^{12}$ & $U^{13}$ & $U^{23}$ \\
\hline C1 & $0.0370(7)$ & $0.0495(8)$ & $0.0482(8)$ & $0.0069(6)$ & $0.0051(6)$ & $-0.0025(6)$ \\
C2 & $0.0360(7)$ & $0.0558(9)$ & $0.0511(8)$ & $0.0084(6)$ & $0.0075(6)$ & $0.0029(7)$ \\
C3 & $0.0396(7)$ & $0.0533(9)$ & $0.0463(8)$ & $0.0093(6)$ & $0.0044(6)$ & $0.0002(7)$ \\
C4 & $0.0325(7)$ & $0.0737(11)$ & $0.0576(9)$ & $0.0083(7)$ & $0.0025(6)$ & $0.0038(8)$ \\
C5 & $0.0358(8)$ & $0.0857(13)$ & $0.0609(10)$ & $0.0011(8)$ & $0.0083(7)$ & $0.0104(9)$ \\
C6 & $0.0391(8)$ & $0.0530(9)$ & $0.0554(9)$ & $0.0111(7)$ & $0.0062(6)$ & $0.0044(7)$ \\
C7 & $0.0503(8)$ & $0.0319(7)$ & $0.0501(8)$ & $0.0043(6)$ & $0.0037(6)$ & $0.0105(6)$ \\
C8 & $0.0658(11)$ & $0.0457(9)$ & $0.0953(15)$ & $0.0063(8)$ & $-0.0247(10)$ & $0.0110(9)$ \\
C9 & $0.0567(12)$ & $0.0702(14)$ & $0.162(3)$ & $0.0015(10)$ & $-0.0182(14)$ & $0.0214(15)$ \\
C10 & $0.0455(8)$ & $0.0638(10)$ & $0.0591(10)$ & $0.0094(7)$ & $0.0124(7)$ & $0.0056(8)$ \\
C11 & $0.0607(11)$ & $0.0857(14)$ & $0.0659(11)$ & $0.0081(10)$ & $0.0104(9)$ & $0.0210(10)$ \\
N1 & $0.0393(7)$ & $0.0669(9)$ & $0.0549(8)$ & $0.0039(6)$ & $0.0054(6)$ & $0.0076(7)$ \\
N2 & $0.0378(6)$ & $0.0494(7)$ & $0.0627(8)$ & $0.0066(5)$ & $0.0027(6)$ & $0.0113(6)$ \\
N3 & $0.0445(7)$ & $0.0314(6)$ & $0.0666(8)$ & $0.0084(5)$ & $-0.0034(6)$ & $0.0054(6)$ \\
O1 & $0.0440(7)$ & $0.1159(12)$ & $0.0797(9)$ & $0.0281(7)$ & $0.0204(6)$ & $0.0354(8)$ \\
O2 & $0.0739(8)$ & $0.0305(6)$ & $0.0743(8)$ & $0.0092(5)$ & $0.0053(6)$ & $0.0045(5)$ \\
O3 & $0.0407(6)$ & $0.0781(8)$ & $0.0612(7)$ & $0.0186(5)$ & $0.0099(5)$ & $0.0205(6)$ \\
& & & & &
\end{tabular}

Geometric parameters $\left(\AA,{ }^{\circ}\right)$

\begin{tabular}{llll}
\hline $\mathrm{C} 1-\mathrm{N} 1$ & $1.330(2)$ & $\mathrm{C} 8-\mathrm{C} 9$ & $1.446(3)$ \\
$\mathrm{C} 1-\mathrm{C} 2$ & $1.383(2)$ & $\mathrm{C} 8-\mathrm{H} 8 \mathrm{~A}$ & 0.9700 \\
$\mathrm{C} 1-\mathrm{C} 6$ & $1.508(2)$ & $\mathrm{C} 8-\mathrm{H} 8 \mathrm{~B}$ & 0.9700 \\
$\mathrm{C} 2-\mathrm{C} 3$ & $1.393(2)$ & $\mathrm{C} 9-\mathrm{H} 9 \mathrm{D}$ & 0.9600 \\
$\mathrm{C} 2-\mathrm{H} 2$ & 0.9300 & $\mathrm{C} 9-\mathrm{H} 9 \mathrm{~A}$ & 0.9600 \\
$\mathrm{C} 3-\mathrm{O} 3$ & $1.3502(19)$ & $\mathrm{C} 9-\mathrm{H} 9 \mathrm{~B}$ & 0.9600 \\
$\mathrm{C} 3-\mathrm{C} 4$ & $1.382(2)$ & $\mathrm{C} 10-\mathrm{O} 3$ & $1.440(2)$ \\
$\mathrm{C} 4-\mathrm{C} 5$ & $1.369(3)$ & $\mathrm{C} 10-\mathrm{C} 11$ & $1.496(3)$ \\
$\mathrm{C} 4-\mathrm{H} 4$ & 0.9300 & $\mathrm{C} 10-\mathrm{H} 10 \mathrm{~A}$ & 0.9700
\end{tabular}




\begin{tabular}{|c|c|c|c|}
\hline $\mathrm{C} 5-\mathrm{N} 1$ & $1.342(2)$ & $\mathrm{C} 10-\mathrm{H} 10 \mathrm{~B}$ & 0.9700 \\
\hline $\mathrm{C} 5-\mathrm{H} 5$ & 0.9300 & $\mathrm{C} 11-\mathrm{H} 11 \mathrm{~A}$ & 0.9600 \\
\hline $\mathrm{C} 6-\mathrm{O} 1$ & $1.212(2)$ & $\mathrm{C} 11-\mathrm{H} 11 \mathrm{~B}$ & 0.9600 \\
\hline $\mathrm{C} 6-\mathrm{N} 2$ & $1.345(2)$ & $\mathrm{C} 11-\mathrm{H} 11 \mathrm{C}$ & 0.9600 \\
\hline $\mathrm{C} 7-\mathrm{O} 2$ & $1.2256(19)$ & $\mathrm{N} 2-\mathrm{N} 3$ & $1.3800(17)$ \\
\hline $\mathrm{C} 7-\mathrm{N} 3$ & $1.335(2)$ & $\mathrm{N} 2-\mathrm{H} 2 \mathrm{~A}$ & 0.8600 \\
\hline $\mathrm{C} 7-\mathrm{C} 8$ & $1.504(2)$ & $\mathrm{N} 3-\mathrm{H} 3 \mathrm{~A}$ & 0.8600 \\
\hline $\mathrm{N} 1-\mathrm{C} 1-\mathrm{C} 2$ & $125.26(14)$ & $\mathrm{C} 8-\mathrm{C} 9-\mathrm{H} 9 \mathrm{D}$ & 109.5 \\
\hline $\mathrm{N} 1-\mathrm{C} 1-\mathrm{C} 6$ & $116.63(14)$ & $\mathrm{C} 8-\mathrm{C} 9-\mathrm{H} 9 \mathrm{~A}$ & 109.5 \\
\hline $\mathrm{C} 2-\mathrm{C} 1-\mathrm{C} 6$ & $118.10(14)$ & $\mathrm{H} 9 \mathrm{D}-\mathrm{C} 9-\mathrm{H} 9 \mathrm{~A}$ & 109.5 \\
\hline $\mathrm{C} 1-\mathrm{C} 2-\mathrm{C} 3$ & $117.38(14)$ & $\mathrm{C} 8-\mathrm{C} 9-\mathrm{H} 9 \mathrm{~B}$ & 109.5 \\
\hline $\mathrm{C} 1-\mathrm{C} 2-\mathrm{H} 2$ & 121.3 & $\mathrm{H} 9 \mathrm{D}-\mathrm{C} 9-\mathrm{H} 9 \mathrm{~B}$ & 109.5 \\
\hline $\mathrm{C} 3-\mathrm{C} 2-\mathrm{H} 2$ & 121.3 & $\mathrm{H} 9 \mathrm{~A}-\mathrm{C} 9-\mathrm{H} 9 \mathrm{~B}$ & 109.5 \\
\hline $\mathrm{O} 3-\mathrm{C} 3-\mathrm{C} 4$ & $116.42(13)$ & $\mathrm{O} 3-\mathrm{C} 10-\mathrm{C} 11$ & $106.39(14)$ \\
\hline $\mathrm{O} 3-\mathrm{C} 3-\mathrm{C} 2$ & $125.11(14)$ & $\mathrm{O} 3-\mathrm{C} 10-\mathrm{H} 10 \mathrm{~A}$ & 110.5 \\
\hline $\mathrm{C} 4-\mathrm{C} 3-\mathrm{C} 2$ & $118.46(15)$ & $\mathrm{C} 11-\mathrm{C} 10-\mathrm{H} 10 \mathrm{~A}$ & 110.5 \\
\hline $\mathrm{C} 5-\mathrm{C} 4-\mathrm{C} 3$ & $119.08(14)$ & $\mathrm{O} 3-\mathrm{C} 10-\mathrm{H} 10 \mathrm{~B}$ & 110.5 \\
\hline $\mathrm{C} 5-\mathrm{C} 4-\mathrm{H} 4$ & 120.5 & $\mathrm{C} 11-\mathrm{C} 10-\mathrm{H} 10 \mathrm{~B}$ & 110.5 \\
\hline $\mathrm{C} 3-\mathrm{C} 4-\mathrm{H} 4$ & 120.5 & $\mathrm{H} 10 \mathrm{~A}-\mathrm{C} 10-\mathrm{H} 10 \mathrm{~B}$ & 108.6 \\
\hline $\mathrm{N} 1-\mathrm{C} 5-\mathrm{C} 4$ & $124.12(16)$ & $\mathrm{C} 10-\mathrm{C} 11-\mathrm{H} 11 \mathrm{~A}$ & 109.5 \\
\hline $\mathrm{N} 1-\mathrm{C} 5-\mathrm{H} 5$ & 117.9 & $\mathrm{C} 10-\mathrm{C} 11-\mathrm{H} 11 \mathrm{~B}$ & 109.5 \\
\hline $\mathrm{C} 4-\mathrm{C} 5-\mathrm{H} 5$ & 117.9 & $\mathrm{H} 11 \mathrm{~A}-\mathrm{C} 11-\mathrm{H} 11 \mathrm{~B}$ & 109.5 \\
\hline $\mathrm{O} 1-\mathrm{C} 6-\mathrm{N} 2$ & $124.08(14)$ & $\mathrm{C} 10-\mathrm{C} 11-\mathrm{H} 11 \mathrm{C}$ & 109.5 \\
\hline $\mathrm{O} 1-\mathrm{C} 6-\mathrm{C} 1$ & $122.24(15)$ & $\mathrm{H} 11 \mathrm{~A}-\mathrm{C} 11-\mathrm{H} 11 \mathrm{C}$ & 109.5 \\
\hline $\mathrm{N} 2-\mathrm{C} 6-\mathrm{C} 1$ & $113.69(14)$ & $\mathrm{H} 11 \mathrm{~B}-\mathrm{C} 11-\mathrm{H} 11 \mathrm{C}$ & 109.5 \\
\hline $\mathrm{O} 2-\mathrm{C} 7-\mathrm{N} 3$ & $122.59(14)$ & $\mathrm{C} 1-\mathrm{N} 1-\mathrm{C} 5$ & $115.67(15)$ \\
\hline $\mathrm{O} 2-\mathrm{C} 7-\mathrm{C} 8$ & $123.14(15)$ & $\mathrm{C} 6-\mathrm{N} 2-\mathrm{N} 3$ & $120.02(13)$ \\
\hline $\mathrm{N} 3-\mathrm{C} 7-\mathrm{C} 8$ & $114.25(13)$ & $\mathrm{C} 6-\mathrm{N} 2-\mathrm{H} 2 \mathrm{~A}$ & 120.0 \\
\hline $\mathrm{C} 9-\mathrm{C} 8-\mathrm{C} 7$ & $116.04(16)$ & $\mathrm{N} 3-\mathrm{N} 2-\mathrm{H} 2 \mathrm{~A}$ & 120.0 \\
\hline $\mathrm{C} 9-\mathrm{C} 8-\mathrm{H} 8 \mathrm{~A}$ & 108.3 & $\mathrm{C} 7-\mathrm{N} 3-\mathrm{N} 2$ & $121.25(12)$ \\
\hline $\mathrm{C} 7-\mathrm{C} 8-\mathrm{H} 8 \mathrm{~A}$ & 108.3 & $\mathrm{C} 7-\mathrm{N} 3-\mathrm{H} 3 \mathrm{~A}$ & 119.4 \\
\hline $\mathrm{C} 9-\mathrm{C} 8-\mathrm{H} 8 \mathrm{~B}$ & 108.3 & $\mathrm{~N} 2-\mathrm{N} 3-\mathrm{H} 3 \mathrm{~A}$ & 119.4 \\
\hline $\mathrm{C} 7-\mathrm{C} 8-\mathrm{H} 8 \mathrm{~B}$ & 108.3 & $\mathrm{C} 3-\mathrm{O} 3-\mathrm{C} 10$ & $118.97(12)$ \\
\hline $\mathrm{H} 8 \mathrm{~A}-\mathrm{C} 8-\mathrm{H} 8 \mathrm{~B}$ & 107.4 & & \\
\hline $\mathrm{N} 1-\mathrm{C} 1-\mathrm{C} 2-\mathrm{C} 3$ & $-1.2(3)$ & $\mathrm{N} 3-\mathrm{C} 7-\mathrm{C} 8-\mathrm{C} 9$ & $-161.1(2)$ \\
\hline $\mathrm{C} 6-\mathrm{C} 1-\mathrm{C} 2-\mathrm{C} 3$ & $178.43(14)$ & $\mathrm{C} 2-\mathrm{C} 1-\mathrm{N} 1-\mathrm{C} 5$ & $0.4(3)$ \\
\hline $\mathrm{C} 1-\mathrm{C} 2-\mathrm{C} 3-\mathrm{O} 3$ & $179.96(15)$ & $\mathrm{C} 6-\mathrm{C} 1-\mathrm{N} 1-\mathrm{C} 5$ & $-179.20(15)$ \\
\hline $\mathrm{C} 1-\mathrm{C} 2-\mathrm{C} 3-\mathrm{C} 4$ & $0.6(2)$ & $\mathrm{C} 4-\mathrm{C} 5-\mathrm{N} 1-\mathrm{C} 1$ & $1.0(3)$ \\
\hline $\mathrm{O} 3-\mathrm{C} 3-\mathrm{C} 4-\mathrm{C} 5$ & $-178.73(17)$ & $\mathrm{O} 1-\mathrm{C} 6-\mathrm{N} 2-\mathrm{N} 3$ & $-5.8(3)$ \\
\hline $\mathrm{C} 2-\mathrm{C} 3-\mathrm{C} 4-\mathrm{C} 5$ & $0.7(3)$ & $\mathrm{C} 1-\mathrm{C} 6-\mathrm{N} 2-\mathrm{N} 3$ & $173.92(13)$ \\
\hline $\mathrm{C} 3-\mathrm{C} 4-\mathrm{C} 5-\mathrm{N} 1$ & $-1.6(3)$ & $\mathrm{O} 2-\mathrm{C} 7-\mathrm{N} 3-\mathrm{N} 2$ & $1.7(2)$ \\
\hline $\mathrm{N} 1-\mathrm{C} 1-\mathrm{C} 6-\mathrm{O} 1$ & $-172.29(17)$ & $\mathrm{C} 8-\mathrm{C} 7-\mathrm{N} 3-\mathrm{N} 2$ & $-179.35(16)$ \\
\hline $\mathrm{C} 2-\mathrm{C} 1-\mathrm{C} 6-\mathrm{O} 1$ & $8.0(3)$ & $\mathrm{C} 6-\mathrm{N} 2-\mathrm{N} 3-\mathrm{C} 7$ & $-77.1(2)$ \\
\hline $\mathrm{N} 1-\mathrm{C} 1-\mathrm{C} 6-\mathrm{N} 2$ & $8.0(2)$ & $\mathrm{C} 4-\mathrm{C} 3-\mathrm{O} 3-\mathrm{C} 10$ & $178.74(16)$ \\
\hline $\mathrm{C} 2-\mathrm{C} 1-\mathrm{C} 6-\mathrm{N} 2$ & $-171.66(14)$ & $\mathrm{C} 2-\mathrm{C} 3-\mathrm{O} 3-\mathrm{C} 10$ & $-0.7(3)$ \\
\hline $\mathrm{O} 2-\mathrm{C} 7-\mathrm{C} 8-\mathrm{C} 9$ & $17.9(3)$ & $\mathrm{C} 11-\mathrm{C} 10-\mathrm{O} 3-\mathrm{C} 3$ & $-177.84(16)$ \\
\hline
\end{tabular}




\section{supporting information}

Hydrogen-bond geometry $\left(A,{ }^{\circ}\right)$

\begin{tabular}{lllll}
\hline$D-\mathrm{H} \cdots A$ & $D-\mathrm{H}$ & $\mathrm{H} \cdots A$ & $D \cdots A$ & $D-\mathrm{H} \cdots A$ \\
\hline $\mathrm{N} 2-\mathrm{H} 2 A \cdots \mathrm{O} 2^{\mathrm{i}}$ & 0.86 & 2.43 & $3.067(2)$ & 132 \\
$\mathrm{~N} 3-\mathrm{H} 3 A \cdots \mathrm{O} 2^{\mathrm{ii}}$ & 0.86 & 2.06 & $2.831(2)$ & 150 \\
\hline
\end{tabular}

Symmetry codes: (i) $-x, y+1 / 2,-z+1 / 2$; (ii) $x, y+1, z$. 